\title{
US ACCELERATOR CONTRIBUTION TO THE LHC*
}

\author{
Michael J. Lamm ${ }^{\#}$, FNAL, Batavia, IL 60510, U.S.A.
}

\begin{abstract}
In 1998, the United States entered into an agreement with CERN to help build the Large Hadron Collider (LHC), with contributions to the accelerator and to the large HEP detectors. To accomplish this, the US LHC Accelerator Project was formed, encompassing expertise from Brookhaven National Laboratory (BNL), Fermi National Accelerator Laboratory (FNAL) and the Lawrence Berkeley National Laboratory (LBNL). This report is a summary of these contributions including the progress towards project completion, as well as a discussion of future plans for continued US participation in the LHC accelerator.
\end{abstract}

\section{OVERVIEW}

The CERN Large Hadron Colider (LHC) is a very large international accelerator construction project whose success depends on contributions from CERN member states and non-member states such as the US. US national laboratories have taken responsibility for providing the final focusing elements for all four LHC experiments as well as other accelerator physics contributions.

The final focusing elements are shown schematically in Fig. 1. Required for this is a set of dipoles, D1 and D2, (D2 not shown) to bring the parallel separated beams in to a single $70 \mathrm{~mm}$ aperture, and an "inner triplet" optical unit $(\mathrm{Q} 1, \mathrm{Q} 2, \mathrm{Q})$ which focuses the beam and provide the final steering into collision. Also required are boxes (DFBX) for distribution of cryogenics, instrumentation, vacuum and magnet current. In the high luminosity interaction regions, absorbers TAS and TAN (not shown) are needed to protect the elements from the significant beam induced heat load generated from interaction region (IR). The US has contributed in several ways. The DFBX boxes are
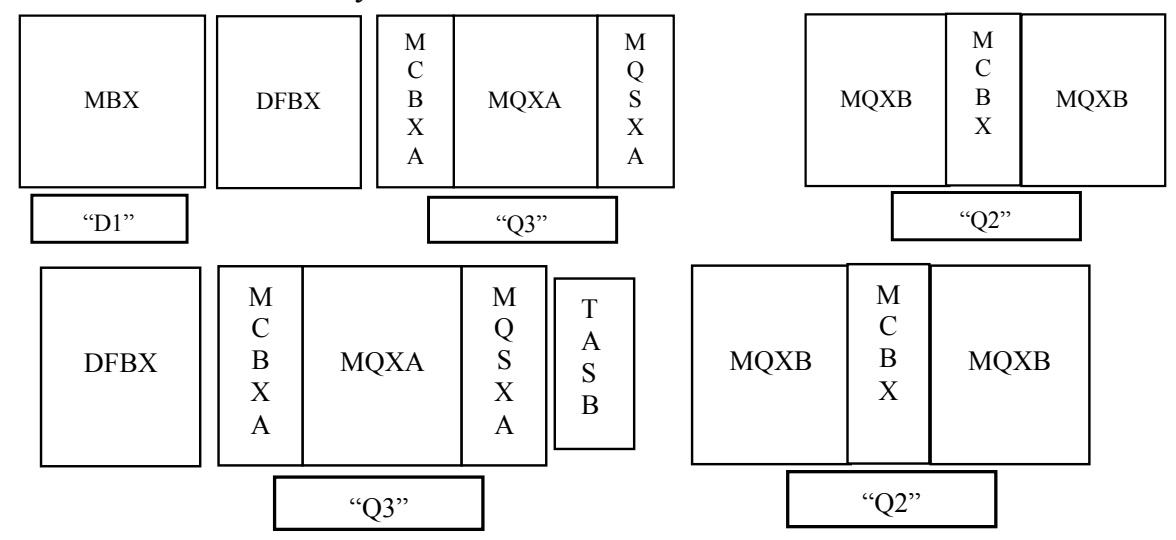

built by a US Vendor with design and oversight from LBNL and FNAL. The TAS/TAN absorbers were built with design and oversight from LBNL. The D2 separation dipole in all 4 IR's and D1 dipole for the low luminosity IR's are supplied by BNL. The Q2 quadrupole elements (MQXB) are designed and built by FNAL, with superconducting cable support from LBNL. The design of the superfluid cryogenic system, quadrupole cryostat and final focus integration were a contribution by FNAL with close collaboration with CERN and the other US institutions. Finally the Q1, Q2, Q3 elements with correctors, buswork and superfluid helium system are assembled at FNAL.

Aside from the final focusing region, there were several other important contributions by the US laboratories. In addition to the D1 and D2, BNL supplied separation dipoles (D3 and D4) for the RF regions. BNL extended its cable testing facilities to aid in the qualification of the LHC superconducting cables. Finally the US accelerator physics groups have made important contributions to the design of the accelerator. Among the projects completed are the specification of the final focus region corrector elements and studies of the energy deposition and alignment tolerances on the final focus magnets.

Before discussing the US contributions in more detail, it is important to acknowledge the significant international contributions to this project. With design and oversight from KEK, Toshiba built the high gradient quadrupoles for the Q1 and Q3 elements (MQXA) [1]. Dipole orbit correctors and correctors for the quadrupole field errors were designed by CERN and built by European vendors [2]. All of the MQXA and corrector magnets have been tested at KEK and CERN prior to shipment to FNAL for integration into the Q1-Q3 elements.

Figure 1. Superconducting magnets and related components for the LHC insertion regions for the low luminosity interaction points (top) and the high luminosity interactions points (bottom).

\footnotetext{
*Work supported by US Department of Energy
}

"lamm@fnal.gov

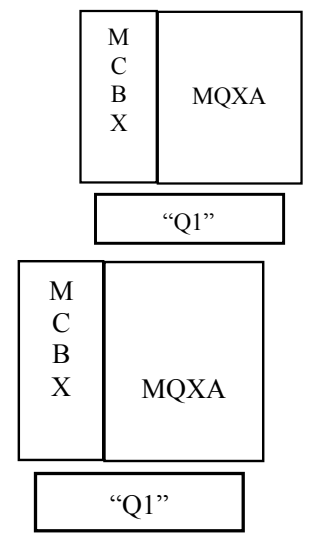




\section{SEPARATION DIPOLES D1-D4}

Twenty dipoles are required for the project which includes four spares. The coils for the D1-D4 magnets have the same design as for RHIC: a single layer $80 \mathrm{~mm}$ aperture dipole made with keystoned $\mathrm{NbTi}$ Rutherford cable. Each element has a magnetic length of $9.45 \mathrm{~m}$. The required peak field is $3.8 \mathrm{~T}$, which can be achieved at $4.5 \mathrm{~K}$ as well as $1.9 \mathrm{~K}$ with a large operating margin. This flexibility with operating temperature made it possible to adapt these dipoles to the needs of other elements in the local cryogenic system. The D1 element is a single aperture magnet which shares its cryogenic system with the $1.9 \mathrm{~K}$ final focus quadrupoles. The D2 and D4 are identical double aperture dipoles with slightly different aperture separation. The D3 consists of two single aperture magnets in a single cryostat. The D2 and D4 share cryostats with adjacent $4.5 \mathrm{~K}$ elements.

Results of the D1-D4 test program are reported elsewhere in this conference [3]. The quench performance of these magnets is very good, with all magnets reaching their operating field with one training quench or less. The measured harmonics are also within accepted limits.

As shown in Table 1, the project is nearly complete, with the majority of the separation dipoles shipped to CERN. Only 5 more dipoles remain at BNL with 2 more tests prior to shipment to CERN.

Table 1: Status of Deliverable Components

\begin{tabular}{cccc}
$\begin{array}{c}\text { US } \\
\text { Component }\end{array}$ & $\begin{array}{c}\text { Required for } \\
\text { Project }\end{array}$ & $\begin{array}{c}\text { Number } \\
\text { Completed }\end{array}$ & $\begin{array}{c}\text { Shipped to } \\
\text { CERN }\end{array}$ \\
\hline DFBX & 8 & 3 & 2 \\
D1 & 5 & 5 & 5 \\
D2 & 9 & 9 & 8 \\
D3 & 3 & 3 & 1 \\
D4 & 3 & 3 & 1 \\
Q1 & 9 & 4 & 4 \\
Q2 & 9 & 6 & 4 \\
Q3 & 9 & 2 & 1 \\
TAS & 4 & 4 & 4 \\
TAN & 4 & 4 & 4
\end{tabular}

\section{INNER TRIPLET FINAL FOCUSING QUADURPOLES}

\section{System Design}

Beams are brought into focus and collision through a "quad triplet" focusing element. Each LHC experimental IR has a matching left-right inner triplet (Q1,Q2,Q3), operating in superfluid. Components for the inner triplet are either built at FNAL or shipped to FNAL for final assembly.

The Q1 and Q3 elements consist of $6.3 \mathrm{~m}$ MQXA quadrupoles from Japan, with corrector elements supplied by CERN. The corrector packages are mechanically attached to the endplates of the MQXA. On the IP end of Q1, there is the required plumbing for the helium return. End domes are welded to the cold mass endplate to complete the helium containment vessel.

The FNAL built Q2 element consists of two $5.5 \mathrm{~m}$ long quadrupoles (MQXB) with a MCBX orbit corrector dipole located between the two MQXB's The two MQXB's are joined together by a stainless steel sleeve, which along with the endplates complete the helium vessel and join the Q2 element static helium bath to the external superfluid heat exchanger.

Prior to welding the ring, the alignment of the two MQXB is checked. Supplemental welding is performed, if necessary, to set the MQXB relative alignment.

The magnets are bussed together using expansion loops and bus bars designed by FNAL. The bus bar stabilizers consist of Rutherford cables of the same dimension as the main conductor. This cable is made of copper, except near the magnet leads of the MQXB where it is a superconducting cable. Tests were performed on these bus bars during the FNAL model program, which showed that these bus bars could be included in the quench protection system of the MQXA and MQXB magnets [4].

Instrumentation and heater wires were specified and largely supplied by CERN. Because of the relative inaccessibility of the final focus elements in the tunnel, particularly the Q1 element, it was decided to bring the instrumentation out through the DFBX. Thus there are 100 wires from the Q1-Q3 elements through the DFBX feedbox. The instrumentation wires are joined in the interconnect region using a connector designed for cryogenic operation.

The heat exchanger system for the inner triplet is modeled after the arc dipoles, i.e. a two phase helium volume in a corrugated copper pipe, surrounded by the static single phase superfluid volume [5]. Unlike the arc dipole and quadrupoles, the superfluid heat exchanger element, shown in Fig. 2, is located outside of the cold mass. It was determined during the design program and verified experimentally [6] that a heater exchanger located inside the cold mass cooling channel would not allow for a copper heater exchange pipe large enough to accommodate the significant heat load which is expected to be $180 \mathrm{~W}$ at full operation luminosity.

The suspension system for this cryostat design uses composite "spider" to support the cold mass and internal piping similar to structures used in the cryogenic transfer lines [7]. These structures are very strong and stiff in the radial direction and make good use of radial space. They also should give small displacements of the geometric magnet axis. These elements do have higher heat loads than the more traditional post designs, but these loads are small compared to the expected $180 \mathrm{~W}$ heat load on the whole system. To better distribute the axial loads to all the support spiders each pair of spiders has a set of axial tie-bars to tie them together. The tie-bars are made from Invar tube to minimize axial forces imposed on the supports during cooldown. The supports are shown in 
Fig. 2. For alignment purposes, the position of the supports can be adjusted through vacuum ports in the outer cryostat vessel (not shown). Finally additional restraints are added prior to shipping to reduce the risk of support damage.

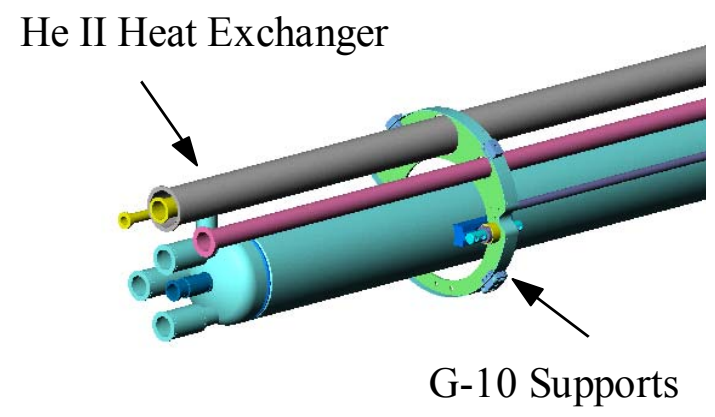

Figure 2. Heat Exchanger and supports for the inner triplet quadrupoles.

\section{MQXB Magnets}

The FNAL-built MQXB is a two layer cos2-theta quadrupole, made from $15 \mathrm{~mm}$ wide $\mathrm{NbTi}$ Rutherford cable. Production details are presented elsewhere [8]. The magnet length is $5.5 \mathrm{~m}$. By bringing out an extra "pigtail" power lead, the two MQXB in each Q2 assembly can be tested separately on our test stand. Test results are present elsewhere [9]. With the exception of two magnets, the training performance of these magnets is very good. In all cases the magnet reached the maximum gradient of $215 \mathrm{~T} / \mathrm{m}$ in one quench or less. On the first few magnets, the magnets went through a thermal cycle, the magnets again reached its operating gradient without a quench.

Two magnets, however reached only $\sim 90$ percent of their required operating gradient. The location of the quenches can be determined through the use of quench antennas. In both cases the quenches occur in the pole region of the inner coil near the lead end. The cause of the quenches is not understood and is under investigation.

The integral field harmonics have been measured for all magnets both at injection and at the peak operating gradient $215 \mathrm{~T} / \mathrm{m}$. They are within accepted values.

\section{Alignment}

Alignment of the inner triplet elements is very important. Transverse tolerances on the order of $0.5 \mathrm{~mm}$ are required in order to have a sufficient dynamic aperture margin. The magnetic axis of each element is measured at room temperature using the "Single Stretched Wire" system developed at FNAL [10]. This axis is related to external fiducials on the cryostat using a Laser Tracker survey system. All of the Q2 elements, and one Q1 and Q3 element are measured at liquid helium temperatures. A typical measurement error in relating the average magnetic axis to the external fiducials is on the order of $0.1 \mathrm{~mm}$. The warm to cold shifts for the Q1 and Q3, measured to be $\sim 0.3 \mathrm{~mm}$, will be applied to the remaining Q1 and Q3 elements.

The Q2 magnets have the additional complication of the relative alignment of the two MQXB elements. While every effort is made to set the relative axes of the individual MQXB to be parallel and within $0.5 \mathrm{~mm}$ of the average magnetic axis, welding of the joining support ring and the thermal contractions of the magnet and support system can change the alignment. Therefore, prior to cold testing, the magnetic axes are measured on the test stand warm and the cryostat supports are adjusted as needed to give the best alignment. During testing in liquid helium the axes are remeasured and then compared to warm alignment measurements after the test. Since the goal is to achieve the best cold alignment, the warm cryostat supports are adjusted to compensate for the measured cold shifts. These adjustments are typically on the order of 0.5 to $1.0 \mathrm{~mm}$.

\section{Status of Final Focus Quadrupoles}

As shown in Table 1, the production of the Q1-Q3 elements is well advanced. With all of the MQXA already delivered to CERN, the Q1 and Q3 elements are proceeding towards completion in early 2006. There are 3 Q2 elements needed for completion. They are in various states of construction, with the expectation of completion also in early 2006.

\section{OTHER ACCLERATOR COMPONENTS}

\section{Cryogenic Feedboxes (DFBX)}

The DFBX distribution boxes are located in line between the Q3 and the D1. Since the final focus quadruple magnets and D1 are identical, these boxes must account for IR to IR differences in magnet and room temperature interfaces, such as low and high luminosity, slope in the accelerator tunnel and left/right mirror image of the power, cryogenic and instrumentation interface.

Each box consists of two or three sets of $7.5 \mathrm{kA}$ power leads to power the final focusing quadrupoles and the D1 dipole in the low luminosity regions. Each pair of leads was tested in a vertical dewar at FNAL. The tests were designed to simulate as much as possible the operating conditions of the LHC. The power leads performed well, each lead was able to pass its acceptance criteria [11].

\section{Hadron and Neutron Absorbers (TAS and TAN)}

At $14 \mathrm{TeV}$ and luminosity of $10^{34} \mathrm{~cm}^{-2} \mathrm{~s}^{-1}$ there will be $\sim 900 \mathrm{~W}$ per beam, largely directed at the inner triplet. In the high luminosity region, two absorbers were built to intercept enough of the energy to allow the final focusing elements to operate with sufficient temperature margin. [12] The TAN (neutral) absorber, meant to intercept forward neutrons and photons, is located in front of the D2 element. It is made of OFHC copper surrounded by an iron box to reduce the exposed surface activation. 
The front hadron absorber TAS consists of a copper beam tube, clam shell copper absorber and an adjustable support and alignment structure. The support system has four adjustable rods which allows for alignment from outside the shielding.

All of these elements have been shipped to CERN.

\section{CABLE TESTING FOR CERN}

For the last two decades, the Cable Test Facility (CTF) at BNL has provided a unique resource for the high energy accelerator community in the development and testing of high current superconducting cables used in accelerator magnets. Work at this facility has supported various accelerator projects during the development and the production phases of magnet construction, including the CBA, SSC, HERA and RHIC and now the LHC.

In the early stages of the program, the facility supported the cable R\&D for the LHC dipole program with the eventual task of providing support for conductor qualification.

To meet the LHC schedule, cable tests were performed on a daily basis for four years. New test stations were built, added on the capability of testing down to $1.8 \mathrm{~K}$, to currents as high as $25 \mathrm{kA}$, in a field as high as $7.5 \mathrm{~T}$ at $4.3 \mathrm{~K}$ and $\sim 9 \mathrm{~T}$ at $1.9 \mathrm{~K}$.

This contribution to the CERN program has been recently completed. After 637 days of testing, 2673 cables were tested, 90 percent of which from the CERN arc magnet program. [13]

\section{ACCELERATOR PHYSICS}

The LHC has also tapped into the wealth of accelerator design and operational experience from the US laboratories. These contributions have centered on providing input into the specification of accelerator components and the optimization of beam operation in the final focus regions. In particular, studies were performed on the effect of the beam from the final focus optics and on the expected magnet heat loads from IR particle production.

Since the beginning of the project there has been good interaction between the magnet builders and the accelerator physicists on correcting and compensating for unwanted field harmonics in order to improve the dynamic aperture. Reference tables were developed for both the MQXA and MQXB from their respective model magnet programs to predict the harmonics of the production magnets. These reference tables were used as input to beam simulation studies to predict dynamic apertures. Accelerator physicists in turn advised the magnet builders on which harmonics were critical to control during production. In the case of the MQXA, this lead to a small modification in the magnet cross section to reduce the b10 harmonic. Studies also showed that the decision to use both MQXA and MQXB magnets in each inner triplet would have a beneficial effect on the dynamic aperture.
The choice of a crossing angle at the high luminosity regions is a trade off between increasing angles to minimize beam-beam interactions and decreasing angles to locate the beam in the good field regions of the inner triplet quadrupoles. Using the MQXA and MQXB harmonics reference tables, studies determined that the crossing angle of 300 micro radians was optimal [14].

In 1999 studies were performed on the local correction of the inner triplets, since it was understood that field errors in the IR collision quadrupoles was the main source of dynamic aperture limitation in the LHC. Using the magnet reference tables, a workshop was organized by BNL with participants from other laboratories to propose a scheme of corrector magnets in the inner triplets. Through collaboration with the LHC Corrector magnet group, the non-linear corrector magnets were developed [15] and included into the final focus elements Q1-Q3.

Luminosity in the LHC will depend critically on the alignment of the inner triplet quadrupoles. These quadrupoles have large gradients and the beam sizes are the largest within these quadrupoles. The absolute alignments of Q1, Q2 and Q3 will be determined during installation. The relative alignment of the two MQXB that make up the Q2 is fixed once they are placed in their common cryostat at Fermilab. A workshop was held at FNAL in October 1999 to study the effect of alignment on the dynamic aperture. As a result of this workshop, an "alignment reference table" was generated as a guideline to the magnet builders and accelerator physicist on the target absolute and relative alignment. Work on the alignment of the Q2 was published in a separate note. [16]

Energy deposition studies performed primarily at FNAL were an important input into the shielding design in the final focus. The shielding consists of the aforementioned TAS and TAN absorbers, as well as a stainless steel absorber between Q2 and Q3, and thick beam tube liners made of stainless steel. Using the MARS code for particle production and energy deposition, it has been shown that the planned absorbers should be sufficient to protect the inner triplet elements [17]. This same group investigated accidental beam aborts due to kicker misfires and proposed ways to protect the magnets around the IR's from such accidents.

\section{FUTURE CONTRIBUTIONS}

With the US LHC construction program coming to a close, plans have already been made for continued US participation in the LHC. The LHC Accelerator Research Program (LARP) collaboration was formed and in the summer of 2003 the baseline program was approved by the Department of Energy.

This program is beneficial to both the US and CERN. CERN could greatly benefit from the US accelerator and magnet expertise. The US will gain experience on this very exciting accelerator program which can be applied to future US initiatives. The net result for the LHC will likely be a more efficient commissioning and startup 
which will result in a better high energy physics program for the international community

The LARP program consists of two related categories (1) accelerator contributions to the commissioning and operation of the LHC and (2) the design and construction of new high field magnets suitable for a luminosity upgrade to the final focus optics.

On the accelerator contributions, discussions between CERN and US personnel are ongoing to identify projects of mutual interests. One task that is already in progress is the installation and commissioning of the aforementioned US deliverables. Other tasks are beam line instrumentation, collimation system and accelerator physics projects related to beam commissioning.

The challenge for the magnet group, which is a collaboration from LBNL, FNAL and BNL, is to produce within the next 5 years a full scale prototype IR quadrupole. Although the specifications for this magnet will be fully developed in the coming years, it is likely that this magnet will require an operating gradient of $>200 \mathrm{~T} / \mathrm{m}$, an aperture of $90 \mathrm{~mm}$ and be able to withstand large energy depositions from the interaction region. These requirements seem to exclude $\mathrm{NbTi}$ even at $1.9 \mathrm{~K}$ and call for magnets built out of Nb3Sn. The first model magnet of this time is being developed this year and should be ready for testing in 2006 .

\section{CONCLUSION}

The US has been given the important responsibility of providing the final focusing elements for the four experimental IR's. After seven years, the US LHC accelerator project is nearing its successful completion. The installation and commissioning of these elements is a part of the US LARP project, which hopes to continue its participation in making the LHC a successful operating accelerator.

\section{ACKNOWLEDGEMENT}

The author would like to acknowledge the staff at BNL, FNAL and LBNL and our KEK and CERN collaborators who participated in the successful US LHC accelerator project, and the management of each laboratory for their support during this project.

The following people contributed plots and information for this paper: Jim Kerby, Tom Nicol, Tom Page, Joe Dimarco, Phil Schlabach, George Velev, Arap Ghosh, Joesph Rasson, Phil Pfund, Joseph Muratore, Sandor Feher, Nikolai Mokhav, Ranko Ostojic, Tom Peterson, Ron Scanlan and Tanaji Sen. A special word of thanks goes to Jim Strait, who was the US Accelerator Project Leader throughout most of this project and to Jim Kerby who is overseeing the project through to completion.

\section{REFERENCES}

[1] A. Yamamoto, Production and Measurement of the MQXA Series of LHC Low-beta Insertion Quadrupoles, ASC 04, Jacksonville, Fl, (2004)

[2] A. Ijspeert et al., "Principles Developed for the Construction of the High Performace Low-Cost Superconducting LHC Corrector Magnets, IEEE Trans. Appl. Supercond., vol 12, No 1. March 2002, pp. $90-93$

[3] J. Muratore et al, MPPT050, this conference.

[4] P. Bauer et al., "Busbar Studies for the LHC Inner Triplet Quadrupoles Built at Fermilab", IEEE Trans. Appl. Supercond., vol 11, No. 1 Mar. 2001, p. 16171620.

[5] R. Byrns et al. "The Cryogenics of the LHC Interaction Region Final Focus Superconducting Magnets", Proceedings of ICEC 17, Bornemouth, England, Jul 1998

[6] C. Darve et al, "A He II Heat Exchanger Test Unit Designed for the LHC Interaction Region Magnets", Adv.Cryog.Eng.43A:403-410,1998

[7] T. Nicol et al, "The LHC Region Cryostat Design and Fabrication", IEEE Trans. Appl. Supercond., Vol 12, No. 1, March 2002, pp. 79-182

[8] R. Bossert et al, "Construction Experience with MQXB Quadrupole Magnets Built at Fermilab for the LHC Interaction Regions", IEEE Trans. Appl. .Supercond. Vol. 13, pp 1297-1300, 2003

[9] G. Velev, "Field Quality Measurements of the LQXB Inner Triplet Quadrupoles for LHC", Presented at ASC 2004, Jacksonville, 2004

[10] J. Dimarco et al, "Field Alignment of Quadrupole Magnets for the LHC Interaction Region", IEEE Trans. Appl. Supercond., Vol. 10, No. 1, March 2000, pp. 127-130

[11] M. Tartaglia et al, "Production LHC HTS Power Lead Test Results", presented at ASC 2004, Jacksonville, 2004

[12] W.C. Turner et al, "Absorbers for the High Luminosity Insertions of the LHC", EPAC 98, Stockholm, pp. 368-370,1998

[13] Arup Ghosh, private communication.

[14] T. Sen, Effect of the Beam-Beam Interactions on the Dynamic Aperture and Amplitude Growth of the LHC , FERMILAB-CONF-99-148, Jun 1999. 8pp.

[15] J. Wie et al, "Interaction Region Local Correction for the Large Hadron Collider", PAC99, New York, 1999. pp. 2921-3

[16] T. Sen et al, "Alignment Tolerance of IR Quadrupoles in the LHC", Fermilab-Conf-99/304, 1999

[17] N.V. Mokhov, "Protecting LHC IP1/IP5 Components Against Radiation Resulting form Colliding Beam I Interactions", FNAL Internal Note FNAL-FN-732 April 2003 